\title{
ON THE CHARACTERISATION OF POLAR FIBROUS COMPOSITES WHEN FIBRES RESIST BENDING
}

\author{
KOSTAS P. SOLDATOS \\ School of Mathematical Sciences, University of Nottingham, Nottingham NG7 2RD, UK \\ and \\ Spencer Institute of Theoretical and Computational Mechanics, University of Nottingham, \\ Nottingham, NG7 2RD, UK
}

In memory of Professor George Simitses

\begin{abstract}
This study aims to initiate research for the invention of methods appropriate for characterisation of fibre-reinforced materials that exhibit polar material behaviour due to fibre bending resistance. It thus focuses interest in the small strain regime, where there are examples of particular deformations for which non-polar linear elasticity fails to distinguish clearly the nature of a fibrous composite or even to account for the presence of fibres. Particular attention is accordingly given to the solution of the polar material version of the pure bending problem of transverse isotropic or special orthotropic plates with embedded fibres resistant in bending. It is seen that pure bending deformation enables polar fibre-reinforced materials to generate constant couple stress-field which, in turn, endorses uniqueness of the solution of the corresponding boundary value problem. In this context, by appropriately extending the validity of Clapeyron's theorem within the regime of polar linear elasticity for fibre-reinforced materials, it is shown that the solution of well-posed linear elasticity boundary value problems that generate a constant couple-stress field is unique. The well-known uniqueness of solution of conventional, non-polar linear elasticity boundary value problems is, in fact, a particular case in which the generated constant value of the couple-stress field is zero.
\end{abstract}


Keywords: Clapeyron's theorem; Fibre-reinforced materials; Fibre bending stiffness; Polar linear elasticity; Pure bending; Orthotropic materials; Transverse isotropic materials; Uniqueness of solution.

\section{Introduction}

When pioneered the non-linear theory of fibre-reinforced materials, Rivlin and his, then, student Adkins assumed deliberately, perhaps for simplicity, that fibres embedded in an isotropic material behave like perfectly flexible cords (Adkins 1951, chps. VIII and X; Adkins and Rivlin, 1955). Their consciousness in making this assumption, which underpinned relevant theoretical developments for long time afterwards (e.g., Pipkin and Rogers, 1971; Spencer, 1972, 1984), becomes clear at the beginning of the Introduction of (Rivlin, 1955).

This assumption is a good approximation in many cases of interest but not applicable when fibres resist bending. In their endeavour to fill in the implied theoretical gap and, hence, complete the earlier theoretical framework, Spencer and Soldatos (2007) developed a non-linear, second gradient, polar hyperelasticity theory that accounts for the bending stiffness of a single family of unidirectional fibres embedded in a relevant composite solid (see also Spencer, 2009). Moreover, Spencer and Soldatos (2007) presented also a corresponding linearised version of that theory, which is accordingly perceived as a polar material completion of conventional linear transverse isotropic elasticity; at least as far as transverse isotropy is due to presence of a single family of unidirectional fibres that resist bending.

It is recalled in this connection that linear anisotropic elasticity has been used extensively in modelling the behaviour of fibre-reinforced solids and structures subjected to small strain after the first half of the $20^{\text {th }}$ century. Nevertheless, interest in and development of linear anisotropic elasticity started much earlier, in the first half of the $19^{\text {th }}$ century (e.g., Love, 1944; Sokolnikoff, 1983). These facts and observations cannot easily dispute a thought that the aforementioned "perfectly flexible fibres" concept pre-existed the early non-linear elasticity models presented by Rivlin and Adkins. 
Most recently, Soldatos $(2014,2015)$ revisited the polar material version of the linearised elasticity theory presented in (Spencer and Soldatos, 2007) and extended it in a manner that accounts for material anisotropy that $(i)$ is due to presence of two families of unidirectional fibres that resist bending, and (ii) may be as advanced as its counterpart met in non-polar locally monoclinic materials. In cases that fibres are perfectly flexible, this new linear anisotropic elasticity development (Soldatos, 2014, 2015) reduces naturally to its conventional counterpart (e.g., Love, 1944; Sokolnikoff, 1983; Ting, 1996; Jones, 1998). However, when fibre bending stiffness is accounted for, the theory faces new challenges which are mainly due to the fact that $(i)$ its governing equations are not anymore elliptic, and (ii) it involves a number of additional elastic moduli whose role is as yet unclear.

In conventional non-polar elasticity, loss of ellipticity of the governing equations may be observed only in the large, non-linear deformation regime, where it is associated with formation of several different modes of possible material instability. Those instability modes manifest themselves as multiple solutions of the relevant non-linear governing equations. That "loss of ellipticity" concept was first investigated by Knowles and Sternberg (1975) within the framework of non-polar isotropic hyperelasticity, while corresponding fibre-reinforced material studies emerged in (Triantafyllidis and Abeyarante, 1983). Research in this subject is still advancing (e.g., Dorfmann et al, 2010; El Hamdaoui and Merodio 2015), but the implied loss of ellipticity concept is already considered the source of different failure modes met in fibre-reinforced material mechanics, including fibre-kinking, fibre de-bonding and fibre splitting (e.g., Merodio and Ogden, 2002; 2003).

In contrast, non-polar linear elasticity guarantees the elliptic nature of its governing equations and, therefore, the uniqueness of the solution of corresponding well-posed boundary value problems; extensive relevant references as well as additional remarks and accounts maybe found, for instance, in (Love, 1944; Sokolnikoff, 1983). In this connection, the fact that the governing equations of the polar linear elasticity presented in (Soldatos 2014; 2015) are not elliptic raises, natural questions and doubts regarding potential uniqueness of the solution of corresponding polar material boundary value problems. Nevertheless, some progress in this direction is still possible, and this is reported in the present study.

On the other hand, the specific role and significance of each of the elastic moduli met in nonpolar isotropic and/or anisotropic linear elasticity has been made clear long ago. This has been achieved by suitable exploitation of the solution of certain fundamental non-polar elasticity boundary 
value problems, such as the unidirectional compression/extension of rods, multi-directional compression/extension, and/or shear loadings of plates, etc. (e.g., Love, 1944; Sokolnikoff, 1983; Jones, 1998). However, the role of the additional elastic moduli entering the polar linear elasticity theory presented in (Soldatos, 2014; 2015) needs still to be investigated and become better understood. An initial effort in that direction is accordingly also presented in this paper, by revisiting and studying in detail the pure bending problem of a fibre-reinforced plate in the light of polar linear elasticity equations stemming from (Soldatos, 2014; 2015).

Under these considerations, Section 2 briefs the basic theoretical background required for this study, including equilibrium equations, basic kinematic and relevant constitutive equations of polar linear anisotropic elasticity. Section 3 introduces next the concepts of the displacement-gradient, the rotation and the spin energy functions. Appropriate use of these concepts enable afterwards Section 4 to develop an interesting polar material extension of Clapeyron's Theorem and, hence, to further prove uniqueness of the solution of a well-posed relevant boundary value problem when the couplestress tensor is constant.

The latter condition guarantees uniqueness of the solution and is used afterwards in the applications considered in Sections 5 and 6. These present a number of useful solutions to different versions of the problem of pure, plane strain bending of an infinitely long fibre-reinforced plate made of perfectly flexible fibres and fibres resistant in bending, respectively. Either of Sections 5 and 6 considers material constitution which is consistent with the material symmetries of both transverse isotropy and special orthotropy. Through comparison of corresponding theoretical results, an initial attempt is thus made towards clarification of the role of the fibre bending stiffnesses met in the polar material version of linear anisotropic elasticity. Basic conclusions as well as relevant thoughts for possible continuation/extension of this study are finally summarised in Section 7.

\section{Basic theory and equations}

Full derivation details of the equations of polar linear elasticity of fibre-reinforced materials may be found in (Soldatos, 2014; 2015). This Section quotes briefly only a set of equations required for the purposes of the present study. Presentation of that set of equations within the framework of a right- 
handed Cartesian co-ordinate system $\mathrm{O} x_{i}$ begins with the introduction of a stress tenor, $\sigma$, and a couple-stress tensor, $\boldsymbol{m}$, which are related as follows:

$\sigma_{i j}=\sigma_{(i j)}+\sigma_{[i j]}, \quad \sigma_{[i j]}=\frac{1}{2} \varepsilon_{k j i} m_{\ell k, \ell}$.

Here $\sigma_{(i j)}$ and $\sigma_{[i j]}$ are the components of the symmetric and the antisymmetric part of the stresstensor, respectively, and $\varepsilon$ is the three-dimensional alternating tensor. Subscripts are generally assumed to take values 1, 2 and 3. Moreover, the summation notation applies on repeated indices and, in the usual manner a comma between indices denotes partial differentiation.

\subsection{Equilibrium and kinematic considerations}

By considering for simplicity that body forces and body couples are absent, a note is initially made of the fact that (2.1b) is essentially an equation that guarantees couple-stress equilibrium in the continuum. Then stress equilibrium considerations yield

$\sigma_{i j, i}=\sigma_{(i j), i}+\frac{1}{2} \varepsilon_{k j i} \bar{m}_{\ell k, \ell i}=0$

where

$\bar{m}_{\ell k}=m_{\ell k}-\frac{1}{3} m_{r r} \delta_{\ell k}$

is the deviatoric part of the couple-stress tensor, and the appearing Kronecker's delta represents the components of the unit matrix, I. Moreover, the components of the traction and the couple-traction vectors acting on any surface $S$ of the fibrous composite are respectively given as follows:

$T_{i}^{(n)}=\sigma_{j i} n_{j}, \quad L_{i}^{(n)}=\bar{m}_{j i} n_{j}$.

where $\boldsymbol{n}$ denotes the outward unit normal of $S$.

It is pointed out with interest that when polar material behaviour manifests itself through generation of a couple-stress field that is at most linear in the spatial co-ordinates, the stress equilibrium equations (2.2) retain the form of their non-polar material counterpart. Moreover, in the particular case that polar material manifestation is associated with generation of a constant couplestress field, then the antisymmetric part of stress (2.1b) is zero and, as a result, the stress field is still symmetric. 
In accordance with conventional, non-polar elasticity kinematics, consider next the displacement vector $\boldsymbol{u}$ and, in the usual manner, define the linear elasticity strain and rotation tensors, $e_{i j}=\frac{1}{2}\left(u_{i, j}+u_{j, i}\right), \omega_{i j}=\frac{1}{2}\left(u_{i, j}-u_{j, i}\right)$.

as the symmetric and the antisymmetric part of the displacement gradient, respectively. Also, recall that the rotation tensor and the spin vector, $\boldsymbol{\Omega}$, are related as follows:

$$
\Omega_{\mathrm{i}}=\frac{1}{2} \varepsilon_{i j k} \omega_{k j}, \omega_{k j}=\varepsilon_{i j k} \Omega_{i}
$$

Assume next that the material of interest contains at most two families of embedded fibres and denote with $\boldsymbol{a}^{(n)}$ the unit vector that defines the direction of the $n$-th family $(n=1,2)$. The curvature vector of either fibre family is defined as the second directional derivative of the displacement vector along the direction of the family, namely

$$
K_{i}^{(n)}=\left(u_{i, k} a_{k}^{(n)}\right)_{, j} a_{j}^{(n)}, \quad(n=1,2) .
$$

For the purposes of this investigation, this curvature vector is considered of the same order of magnitude with the first-order displacement gradients and, therefore, with the strain and the rotation components (2.5).

\subsection{Constitution}

In accordance with linear elasticity postulates, consider next that the material possesses a strain energy function, $W$, which is $(i)$ positive definite and (ii) strictly quadratic in $u_{i, j}$ and $K_{i}^{(n)}$. It can then be shown that $W$ is necessarily of the form

$$
W\left(\mathbf{e}, \mathbf{K}^{(n)} ; \mathbf{a}^{(n)}\right)=W^{e}\left(\mathbf{e} ; \mathbf{a}^{(1)}, \mathbf{a}^{(2)}\right)+W^{K}\left(\mathbf{K}^{(1)}, \mathbf{K}^{(2)} ; \mathbf{a}^{(1)}, \mathbf{a}^{(2)}\right),
$$

where $W^{e}$ is identical to its conventional, non-polar linear elasticity counterpart and is, therefore, positive definite. On the other hand, general forms of $W^{K}$ are detailed in (Soldatos, 2015) up to the material symmetry of a locally monoclinic material. Any of those forms is required to be positive semidefinite.

It follows that,

$$
\sigma_{(i j)}=\frac{\partial W^{e}}{\partial e_{i j}}=c_{i j k l} e_{k l}, W^{e}\left(e_{i j}\right)=\frac{1}{2} \sigma_{(i j)} e_{i j}=\frac{1}{2} c_{i j k l} e_{k l} e_{i j},
$$


where the components of the appearing fourth-order tensor, $\boldsymbol{c}$, obey the symmetry relationships (e.g., Ting, 1996; Jones, 1998)

$c_{i j k l}=c_{j i k l}=c_{i j l k}=c_{k l i j}$.

These relationships enable thus (2.9a) to obtain the well-known alternative form

$$
\left[\begin{array}{l}
\sigma_{11} \\
\sigma_{22} \\
\sigma_{33} \\
\sigma_{(23)} \\
\sigma_{(13)} \\
\sigma_{(12)}
\end{array}\right]=\left[\begin{array}{llllll}
C_{11} & C_{12} & C_{13} & C_{14} & C_{15} & C_{16} \\
C_{12} & C_{22} & C_{23} & C_{24} & C_{25} & C_{26} \\
C_{13} & C_{23} & C_{33} & C_{34} & C_{13} & C_{36} \\
C_{14} & C_{24} & C_{34} & C_{44} & C_{45} & C_{46} \\
C_{15} & C_{25} & C_{35} & C_{45} & C_{55} & C_{56} \\
C_{16} & C_{12} & C_{13} & C_{14} & C_{15} & C_{66}
\end{array}\right]\left[\begin{array}{l}
e_{11} \\
e_{22} \\
e_{33} \\
2 e_{23} \\
2 e_{13} \\
2 e_{12}
\end{array}\right],
$$

where, explicit connections between the components of the matrix $[C]$ and those of the tensor $c$ are also well-known. It is recalled that several symmetries of specific material anisotropy can be established by considering that fibres attain specific corresponding directions within the adopted Cartesian co-ordinate framework (e.g., Ting, 1996; Jones, 1998). In each case, the values of a relevant group of elastic moduli are shown equal to zero and, hence, enable the elastic stiffness matrix $[C]$ to obtain a form that is simpler to its counterpart shown in (2.11).

It has been shown on the other hand (Soldatos, 2015) that the general form of a constitutive equation for the couple-stress tensor is as follows:

$$
\bar{m}_{\ell r}=\sum_{n=1}^{2} \bar{m}_{\ell r}^{(n)}=\frac{4}{3} \varepsilon_{r s i} \sum_{n=1}^{2} \frac{\partial W^{K}}{\partial K_{i}^{(n)}} a_{s}^{(n)} a_{\ell}^{(n)} .
$$

Nevertheless, particular forms of these constitutive equations are also detailed in (Soldatos, 2015) for symmetries of material anisotropy that progressively approach the symmetry of a locally monoclinic material. However, it is sufficient for the purposes of the present study to consider only forms that these constitutive equations obtain in particular cases of transverse isotropy and othotropy.

Accordingly, transverse isotropy due to the presence of a single family of straight fibres parallel to the $x_{1}$-direction is specified by choosing $n=1$ and $\boldsymbol{a} \equiv \boldsymbol{a}^{(1)}=(1,0,0)^{T}$. In that case, the nonzero components of the couple-stress tensor (2.3) may be given explicitly as follows:

$$
\bar{m}_{12}=-d^{f} u_{3,11}, \quad \bar{m}_{13}=d^{f} u_{2,11} \text {. }
$$

Similarly, if transverse isotropy is due the presence of straight fibres parallel to the $x_{2}$-direction, then $\boldsymbol{a} \equiv \boldsymbol{a}^{(2)}=(0,1,0)^{T}$ and the corresponding non-zero couple-stress components are $\bar{m}_{21}=d^{f} u_{3,22}, \quad \bar{m}_{23}=-d^{f} u_{1,22}$. 
Unlike the components of the aforementioned matrix $[C]$ which have dimensions of stress, the single elastic modulus appearing in (2.13), namely $d^{f}$, has the dimensions of force. This modulus relates linearly the appearing non-zero couple-stress components with corresponding components of the fibre curvature vector (also termed as curvature-strains) and is accordingly identified as the elastic modulus of fibre bending resistance or, equivalently, as the fibre bending stiffness.

A corresponding case of orthotropy is characterised by two mutually orthogonal families of fibres embedded in the fibrous composite of interest $(n=2)$. If the fibres of either family are straight, their directions may be considered parallel to the $x_{1}$ - and $x_{2}$-directions by choosing $\boldsymbol{a}^{(1)}=(1,0,0)^{T}$ and $\boldsymbol{a}^{(2)}=(0,1,0)^{T}$. In that case of so-called special orthotropy, the non-zero components of the couplestress tensor (2.3) are given in terms of the curvature-strains according as follows:

$$
\left[\begin{array}{c}
-\bar{m}_{12} \\
\bar{m}_{21} \\
\bar{m}_{13} \\
-\bar{m}_{23}
\end{array}\right]=\left[\begin{array}{cccccc}
d_{11} & d_{12} & 0 & 0 & 0 & 0 \\
d_{12} d_{22} & 0 & 0 & 0 & 0 \\
0 & 0 & d_{31} & d_{32} & 0 & 0 \\
0 & 0 & 0 & 0 & d_{13} & d_{23}
\end{array}\right]\left[\begin{array}{l}
u_{3,11} \\
u_{3,22} \\
u_{2,11} \\
u_{2,22} \\
u_{1,11} \\
u_{1,22}
\end{array}\right] .
$$

These involve seven independent elastic moduli of fibre bending resistance, all of which have evidently the dimensions of force.

Unlike $d^{f}$, which represents the fibre bending resistance of a single family of unidirectional fibres in (2.13), each one of the seven elastic moduli appearing in the matrix $[d]$ above is generally regarded as superposition of corresponding parameters that represent different modes of fibre bending resistance within the orthotropic material of interest. Some of these modes refer to bending resistance of a single fibre family only, while others reflect coupled bending resistance of both families (Soldatos, 2015).

\section{The displacement-gradient, the rotation and the spin energy functions}

It is well-known that the solution of a well-posed non-polar linear elasticity boundary value problem is unique, and that this minimises the potential energy of the relevant deformation. As a result, 
minimisation of the potential energy of the deformation, for instance through careful application of the Ritz method, consists one of the most popular solution methods to complicated non-polar linear elasticity boundary value problems.

The extent to which these features may be still applicable to corresponding polar linear elasticity problems of fibre-reinforced materials and structures is currently unknown. It is, however, already known (Soldatos, 2014; 2015) that, due to the non-elliptic nature of the relevant governing equations, certain, so-called weak discontinuity solutions may be present. Such polar elasticity solutions are represented by displacements which are continuous throughout the fibrous composite of interest but possess first-, second- or higher-order derivatives which may be discontinuous on certain material surfaces.

Weak discontinuity solutions may co-exist with possible solutions formed by continuous displacements that also possess continuous derivatives of all orders throughout the body of the same fibrous composite. Their existence raises thus natural doubts on the extent that anyone's attempt to prove uniqueness of the solution of a general well-posed mixed boundary value problem in polar linear elasticity of fibre-reinforced materials might be successful. Nevertheless, relevant progress is still possible in this case, at least by tracing the extent to which a couple of classical theorems met in non-polar linear elasticity can still be useful.

To this end, and in a close connection with the definition of the strain energy function (2.9b), the displacement-gradient energy function is here defined as follows:

$U\left(e_{i j}\right)=\frac{1}{2} \sigma_{j i} u_{i, j}$.

With use of (2.1) and (2.5), this is seen equivalent to

$U\left(e_{i j}\right)=\frac{1}{2}\left(\sigma_{(j i)}+\sigma_{[j i]}\right)\left(e_{i j}+\omega_{i j}\right)=\frac{1}{2}\left(\sigma_{(i j)} e_{i j}+\sigma_{[j i]} \omega_{i j}\right)=W^{e}\left(e_{i j}\right)+W^{\omega}\left(\omega_{i j}\right)$,

where use is also made of (2.9b), and the appearing rotation energy function is defined as follows: $W^{\omega}\left(\omega_{i j}\right)=\frac{1}{2} \sigma_{[j i]} \omega_{i j}$.

By virtue of (2.1) and (2.6b), one obtains further

$W^{\omega}\left(\omega_{i j}\right)=\frac{1}{2} \varepsilon_{k i j} m_{\ell k, \ell} \omega_{i j}$,

and then 


$$
W^{\omega}\left(\omega_{i j}\right)=\frac{1}{2} \varepsilon_{k i j}\left(m_{\ell k} \omega_{i j}\right)_{, \ell}-\frac{1}{2} \varepsilon_{k i j}\left(\bar{m}_{\ell k}+\frac{1}{3} m_{r r} \delta_{\ell k}\right) \omega_{i j, \ell}
$$

where use is made of the product rule of differentiation.

Nevertheless, use of (2.5b) reveals that $\varepsilon_{k i j} \delta_{\ell k} \omega_{i j, \ell}=\varepsilon_{k i j} \omega_{i j, k}=0$ in (3.5), and, as a result, the rotation energy function obtains the alternative form

$$
W^{\omega}\left(\omega_{i j}\right)=W^{\Omega}\left(\Omega_{i}\right)-\left(m_{i j} \Omega_{j}\right)_{, i}
$$

where the appearing spin energy function is defined as follows:

$W^{\Omega}\left(\Omega_{i}\right)=\bar{m}_{i j} \Omega_{j, i}$.

It is thus seen that the displacement-gradient energy function (3.2) may be written as the following sum of terms:

$U\left(e_{i j}\right)=W^{e}\left(e_{i j}\right)+W^{\Omega}\left(\Omega_{i}\right)-\left(m_{i j} \Omega_{j}\right)_{, i}$,

the first of which is the standard strain energy function met in non-polar linear elasticity. The second term, namely the spin energy function (3.7), is due to action of the curvature-strains and is quadratic in the latter. Finally, the last term appearing in the right hand side of (3.8) will be seen next relevant to non-zero couple-tractions that may possibly be applied externally on the bounding surface of the polar fibre-reinforced material of interest.

\section{Two fundamental theorems in polar linear elasticity of fibre-reinforced materials}

\section{Theorem 1:}

If a polar fibre-reinforced elastic body of volume $V$ is in equilibrium under the action of tractions $\boldsymbol{T}$ and couple-tractions $\boldsymbol{L}$ applied externally on its bounding surface $S$, then the sum of the strain and spin energies of deformation equals the sum of one half of the work done by the external forces and the work done by the external moments acting through their ultimate displacement and spin fields, $\boldsymbol{u}$ and $\boldsymbol{\Omega}$, respectively.

\section{Proof:}

Multiplying both sides of (2.2) by the displacement vector $\boldsymbol{u}$ and, then, integrating the result over the volume $V$, one obtains 
$\int_{V} \sigma_{j i, j} u_{i} d V=0$

or, equivalently,

$\int_{V}\left\{\left(\sigma_{j i} u_{i}\right)_{, j}-\sigma_{j i} u_{i, j}\right\} d V=0$.

Applying the divergence theorem on the first term of the integrant and making use of (3.1), one obtains next

$\int_{S} n_{j} \sigma_{j i} u_{i} d S-2 \int_{V} U\left(e_{i j}\right) d V=0$,

which, with further use of (2.4a) and (3.8), is seen equivalent to

$\int_{S} T_{i} u_{i} d S=2 \int_{V}\left[W^{e}\left(e_{i j}\right)+W^{\Omega}\left(\Omega_{i}\right)-\left(m_{i j} \Omega_{j}\right)_{, i}\right\rfloor d V$.

Application of the divergence theorem on the third term of the volume integral yields

$\int_{S} T_{i} u_{i} d S=2 \int_{V}\left[W^{e}\left(e_{i j}\right)+W^{\Omega}\left(\Omega_{i}\right)\right] d V-2 \int_{S} n_{i} m_{i j} \Omega_{j} d S=0$.

Hence, use of (2.4b) leads to

$\int_{S}\left(T_{i} u_{i}+2 L_{i} \Omega_{i}\right) d S=2 \int_{V}\left[W^{e}\left(e_{i j}\right)+W^{\Omega}\left(\Omega_{i}\right)\right] d V$,

from which the theorem follows.

It is noted that, by virtue of (3.4), if a polar fibre-reinforced material is deformed under the action of a constant couple-stress field, then the value of the rotation energy function is zero. In that case, (3.6) reveals that

$W^{\Omega}\left(\Omega_{i}\right)=\left(m_{i j} \Omega_{j}\right)_{, i}$,

and because corresponding terms cancel thus each other in (4.3), the latter equation or, equivalently (4.5) reduces to

$\int_{S} T_{i} u_{i} d S=2 \int_{V} W^{e}\left(e_{i j}\right) d V$.

This particular form of (4.5) is well known in non-polar linear elasticity and concludes the proof of the non-polar counterpart of Theorem 1 which is attributed to Clapeyron (e.g., Solonlikoff, 1983, p. 86). 
In view of the fact, that Clapeyron's theorem underpins the proof of the uniqueness of wellposed boundary value problems in non-polar linear elasticity, one observes that the following, more general uniqueness theorem holds true in the present case of interest:

\section{Theorem 2:}

The solution of a well-posed boundary value problem in linear elasticity of fibre-reinforced materials is unique if the generated couple-stress field is constant.

\section{Proof}

If the generated couple-stress field is constant, then the rotation energy function is zero by virtue of (3.4). Hence, validity of (3.6) leads to (4.6) and, as a result, (4.3) reduces to (4.7). The proof of this theorem is thus reduced to the proof of its non-polar elasticity counterpart (e.g., Sokolnikoff, 1983).

It is thus seen that the corresponding non-polar linear elasticity theorem is a particular case of Theorem 2 above, in the sense that, in the particular case of non-polar elasticity, the implied constant value of the couple-stress field is zero. Hence, the solution uniqueness of any well-posed problem solved already in non-polar linear elasticity falls into the regime of applicability of Theorem 2 above. The relatively simple linear elasticity problems discussed in the next couple of Sections do meet the conditions of Theorem 2 and, hence, fall into its regime of applicability as well.

In this context, it is finally worth noting that in polar elasticity boundary value problems in which the couple-stress tensor is constant and, hence, Theorem 2 applies, (2.1) reveals that the stress tensor is still symmetric. Thus, the equilibrium equation (2.2) still obtains its conventional, non-polar elasticity form in such cases.

Nevertheless, it is evident that (2.2) may still obtain its form met in non-polar elasticity even in cases of boundary value problems in which the couple-stress field varies linearly in the spatial coordinate parameters. This happens despite the fact that, in general, neither the stress field is symmetric nor the value of the rotation energy function is zero in this type of boundary value problems, where no information is currently available regarding their solution uniqueness. 


\section{Pure, plane strain bending of a non-polar, infinitely long fibre-reinforced plate}

The formulation, as well as the solution of the classical problem of pure, plane strain bending of a rectangular, homogeneous isotropic plate of infinite length is attributed to Saint-Venant, at the era of the early research developments in non-polar linear elasticity (see Love, 1944, p.129). The crosssection of such a rectangular elastic plate, having width $L$ in the $x_{1}$-direction and thickness $h$ in the $x_{2}$ direction is shown in Figure 1.

The plate is long in the $x_{3}$-direction, which is normal to plane of the Figure, to an extent that enables someone to consider that its length is infinite. The ends $x_{1}= \pm L / 2$ of every cross-section are free from shear tractions but are subjected to the externally applied normal stress distribution $\sigma_{11}=\hat{\sigma}_{1} x_{2}$

where $\hat{\sigma}_{1}$ is a known positive constant. The ends $x_{2}= \pm h / 2$ are traction free.

It is a relatively simple matter for someone to show that if (5.1) and $\sigma_{12} \equiv \sigma_{(12)}=\sigma_{22}=0, \quad \sigma_{[12]} \equiv 0$,

are adopted to represent the stress distribution throughout the cross-section of the plate, then the implied all around traction boundary conditions and the corresponding equilibrium equations, namely (2.2) with $\boldsymbol{m}=\mathbf{0}$, are satisfied identically in the case of non-polar linear elasticity.

It follows that (5.1) and (5.2) represent the stress distribution associated with the unique, exact solution of the described non-polar linear elasticity boundary value problem, provided that a corresponding displacement field does exist and is found. That displacement field becomes evidently available in non-polar isotropic elasticity (e.g., Love, 1944; Timoshenko and Goodier, 1970) by using the inverse of Hooke's law and, then, integrating appropriately the resulting strain components.

Nevertheless, (5.1) and (5.2) represent the stress distribution associated with the unique, exact solution of this non-polar linear elasticity boundary value problem regardless of either the type or the cause of the observed material anisotropy. In this regard, the displacement field associated with the stress distribution (5.1) and (5.2) is next sought and found for the most general case of possible anisotropy, where the plane strain version of the generalised Hooke's law (2.11) takes the form

$$
\left[\begin{array}{l}
\sigma_{11} \\
\sigma_{22} \\
\sigma_{(12)}
\end{array}\right]=\left[\begin{array}{lll}
C_{11} & C_{12} & C_{16} \\
C_{12} & C_{22} & C_{26} \\
C_{16} & C_{26} & C_{66}
\end{array}\right]\left[\begin{array}{l}
e_{11} \\
e_{22} \\
2 e_{12}
\end{array}\right] .
$$


For mathematical convenience, this constitutive equation is rearranged as follows:

$\left[\begin{array}{l}e_{11} \\ e_{22} \\ 2 e_{12}\end{array}\right]=\left[\begin{array}{lll}S_{11} & S_{12} & S_{16} \\ S_{12} & S_{22} & S_{26} \\ S_{16} & S_{26} & S_{66}\end{array}\right]\left[\begin{array}{l}\sigma_{11} \\ \sigma_{22} \\ \sigma_{(12)}\end{array}\right]$,

where the matrix of the elastic compliances, $[S]$, is evidently the inverse of the plane strain version of the elastic stiffness matrix $[C]$ appearing in (5.3) (e.g., Jones, 1998).

By inserting the stress field (5.1) and (5.2) into (5.4), and using the definition of the strain tensor (2.5a), one obtains the following set of simultaneous partial differential equations for the pair of the in-plane unknown displacement components $\left(u_{3}=0\right)$ :

$u_{1,1}=S_{11} \hat{\sigma}_{1} x_{2}, \quad u_{2,2}=S_{12} \hat{\sigma}_{1} x_{2}, u_{1,2}+u_{2,1}=S_{16} \hat{\sigma}_{1} x_{2}$.

Integrating this set of equations and, then, ignoring rigid body translation, one obtains with relative ease the in-plane displacement components sought as follows:

$u_{1}=\hat{\sigma}_{1} x_{2}\left(S_{11} x_{1}+\frac{1}{2} S_{16} x_{2}\right), u_{2}=\frac{1}{2} \hat{\sigma}_{1}\left(S_{12} x_{2}^{2}-S_{11} x_{1}^{2}\right)$,

where care is also taken for the rotation $\omega_{12}$ to vanish at the co-ordinate origin.

An estimate of the resulting curvature of an element parallel to the $x_{1}$-axis is thus

$\kappa_{1} \equiv\left|K_{1}^{(1)}\right|, \quad K_{1}^{(1)}=u_{2,11}=-S_{11} \hat{\sigma}_{1}$,

where, for an initial connection with the corresponding polar elasticity problem (see Section 6 below), use is also made of the curvature-strain terminology employed in (2.7). Within the regime of validity of linear elasticity, (5.7) provides a good and acceptable approximation of the actual curvature of the horizontal element or, in the case of a very thick plate, of the average relevant curvature (e.g., curvature of cross-sectional middle-axis).

The resultant bending moment per unit width of the plate has magnitude

$M_{3}=\left.\int_{-h / 2}^{h / 2} \sigma_{11}\right|_{x_{1}= \pm L} x_{2} d x_{2}=\frac{h^{3}}{12} \hat{\sigma}_{1}=\frac{h^{3}}{12 S_{11}} \kappa_{1}$,

and, in accordance with the sign of $(5.7 \mathrm{~b})$, is directed along the positive $x_{3}$-axis. In agreement with the relevant, well-known Euler-Bernoulli rule, this moment is proportional to the resulting middle-axis curvature, $\kappa_{1}$.

It is re-emphasised that the outlined solution and subsequent relevant results apply regardless of whether the general material anisotropy considered is due to fibre presence. However, interest will 
next focus on anisotropic material behaviour caused by the presence of either a single family of straight fibres or a pair of such families. Within this framework of interest, the displacement distributions (5.6) refers to material fibre reinforcement caused by two dissimilar families of perfectly flexible straight fibres. If these families are non-orthogonal, then the induced type of anisotropy is that of a so-called monoclinic material, while the case of two mutually orthogonal fibre families endorses generally orthotropic behaviour.

In the case of special orthotropy, where the direction of the mutually orthogonal families of perfectly flexible fibres coincides with the $x_{1}$ - and $x_{2}$-directions, it is $S_{16}=S_{26}=C_{16}=C_{26}=0$ and, hence, (5.6a) obtains a slightly simplified form. When expressed in terms of the non-zero elastic stiffness moduli, the relevant displacement field is thus found to be

$u_{1}=\frac{\hat{\sigma}_{1} C_{22} x_{1} x_{2}}{C_{11} C_{22}-C_{12}^{2}}, u_{2}=-\frac{\hat{\sigma}_{1}\left(C_{22} x_{1}^{2}+C_{12} x_{2}^{2}\right)}{2\left(C_{11} C_{22}-C_{12}^{2}\right)}$,

while the formulas that correspond to (5.7) and (5.8) take the form

$\kappa_{1} \equiv\left|K_{1}^{(1)}\right|=\frac{C_{22} \hat{\sigma}_{1}}{C_{11} C_{22}-C_{12}^{2}}, \quad M_{3}=\frac{h^{3}}{12} \hat{\sigma}_{1}=\frac{h^{3}\left(C_{11} C_{22}-C_{12}^{2}\right)}{12 C_{22}} \kappa_{1}$,

respectively.

It is pointed out that (5.9) and (5.10) remain unchanged in the case of transverse isotropy caused by the presence of a single family of perfectly flexible straight fibres oriented along either the $x_{1}$ - or the $x_{2}$-direction. It is thus observed that, as far as this pure bending problem and its solution are concerned, non-polar linear elasticity makes no distinction between the implied cases of transverse isotopy and special orthotropy.

In contrast, the analysis presented in Section 6 below will make evident that non-polar elasticity considerations make a clear distinction between these two different special cases of general material anisotropy. It is recalled in this context that another similar case, where non-polar linear elasticity fails to even account for the presence of fibres has previously been detected in (Dagher and Soldatos, 2011).

A further reduction of (5.9) and (5.10) is evidently possible in the case of material isotropy. With the fibres absent in that case, and, hence, by virtue of $C_{11}=C_{22}=\frac{(1-v) E}{(1+v)(1-2 v)}, \quad C_{12}=\frac{v E}{(1+v)(1-2 v)}$, 
the outlined solution and relevant analysis reduce naturally into their corresponding non-polar isotropic elasticity counterparts (Love, 1944; Timoshenko and Goodier, 1970). Here, $E$ and $v$ represent the Young's modulus and the Poisson ratio, respectively.

It is further noted with interest that, unlike (5.6), the displacement distribution (5.9) yields $e_{12}$ $=0$. It follows that, as far as the aforementioned material classes of transverse isotropy, special orthotropy and isotropy are concerned, material elements which are initially parallel to the $x_{2}$-axis remain approximately straight and normal to the deformed $x_{1}$-axis. Alternatively, this observation reveals that (5.9) and the associated exact elasticity analysis relate to applied mechanics problems in which material elements that are initially parallel to the $x_{2}$-axis may approximately remain $(i)$ straight after deformation, and (ii) normal to the deformed $x_{1}$-axis which is bent in the manner suggested by (5.10).

It is recalled in this context that there exist in the mechanics and structural analysis literature a plethora of problems where the accuracy of (5.10) is considered undisputable, particularly when the thickness parameter depicted in Figure 1 is much smaller than its axial counterpart $(h<<L)$. For example, the outlined observations are all consistent with the principal hypotheses that the so-called "classical" models of thin-walled beams, plates and shells are based on (e.g., Kirchhoff, 1850; Reissner and Stavsky, 1961; Donnell, 1976). It is, in fact, precisely those hypotheses which are reconsidered in cases that structural dimensions, loading conditions, and/or special material properties require development/use of refined and more accurate thin-walled structure modelling (e.g., Timoshenko, 1921, 1922; Reissner, 1945; Mindlin, 1951; Bhimaraddi and Stephens, 1984; Reddy, 1984; Soldatos and Watson, 1997; Soldatos, 2003).

\section{Pure bending of a corresponding polar fibre-reinforced plate}

In the case that the same problem is studied in the light of polar elasticity considerations $(\boldsymbol{m} \neq \mathbf{0})$, validity of (5.2a), namely $\sigma_{12}=0$ and $\sigma_{21}=0$, implies that still is

$$
\sigma_{(12)}=\sigma_{[12]}=0
$$

throughout the plate cross-section. Because equation (5.3) or, equivalently, (5.4) is still consistent with the symmetric part of the stress state, the analysis presented in the preceding section still holds. 
The polar material cases considered in this Section are those of transverse isotropy due to the presence of a single family of straight fibres oriented along either the $x_{1}$ - or the $x_{2}$-direction, and that of the special orthoropy detailed above. In all three cases, it is $S_{16}=S_{26}=C_{16}=C_{16}=0$ and, hence, the stress constitutive equation (4.3) obtains the simplified form

$$
\left[\begin{array}{l}
\sigma_{11} \\
\sigma_{22} \\
\sigma_{(12)}
\end{array}\right]=\left[\begin{array}{ccc}
C_{11} & C_{12} & 0 \\
C_{12} & C_{22} & 0 \\
0 & 0 & C_{66}
\end{array}\right]\left[\begin{array}{l}
e_{11} \\
e_{22} \\
2 e_{12}
\end{array}\right] .
$$

As is already mentioned, non-polar elasticity makes thus no direct mathematical distinction between special orthotropy and either of the implied transverse isotopy cases, in the sense that, in all three cases the corresponding non-zero displacement components are those given in (5.9).

Nevertheless, the relevant couple-stress field should now be also accounted for, by considering the appropriate form of a corresponding constitutive equation, namely (2.13) or (2.14). Because plane strain requirements impose the condition $u_{3}=0$, curvature-strain components that may need to be considered in those equations are only the ones related with the non-zero expressions of $u_{1}$ and $u_{2}$. According to (5.9), those curvature-strains are

$$
u_{2,11}=-\hat{\sigma}_{1} S_{11}, \quad u_{2,12}=0, u_{2,22}=\hat{\sigma}_{1} S_{12}, u_{1,11}=0, u_{1,12}=\hat{\sigma}_{1} S_{11}, u_{1,22}=0 .
$$

Hence, (2.13) and (2.14) make clear that $\bar{m}_{13}$ and $\bar{m}_{23}$ may be the only non-zero couple-stress components that enter the present plane strain analysis.

It is observed that the curvature-strains (6.3) are all constant and, hence, the resulting non-zero couple-stress components will also be constant. It follows that the polar linear elasticity boundary value problems considered in this Section fall into the regime of applicability of Theorem 2 above (see Section 4) and their discussed solution is accordingly unique.

\subsection{Transverse isotropy}

When fibres resist bending, polar elasticity is content with (5.9) and (5.10) only in the case that transverse isotropy is due to straight fibres oriented along the $x_{2}$-direction. This is because, as (6.3f) reveals, fibres parallel to the $x_{2}$-axis do not bent under the loading conditions depicted in Figure 1. Accordingly, (2.13b) predicts that all couple-stress components are zero and as a result, the plate responds in a non-polar material manner. 
If, on the other hand, a single family of fibres that resist bending is placed parallel to the $x_{1}$ axis, then the displacements (5.9) produce only a single nonzero couple-stress component, namely

$$
\bar{m}_{13}=-d^{f} \hat{\sigma}_{1} S_{11}=-\frac{d^{f} C_{22} \hat{\sigma}_{1}}{C_{11} C_{22}-C_{12}^{2}}=-d^{f} \kappa_{1}=-\hat{m}_{3},
$$

where the positive constant $\hat{m}_{3}$ is introduced to represent its intensity. Consideration of (2.4b) reveals further that, in this case, the following sets of boundary couple-tractions need to also be taken into consideration:

at $x_{1}= \pm L / 2: L_{3}^{\left(e_{1}\right)}=-\hat{m}_{3}, L_{1}^{\left(e_{1}\right)}=L_{2}^{\left(e_{1}\right)}=0$,

at $x_{2}= \pm h / 2: L_{i}^{\left(e_{2}\right)}=0$,

where $\boldsymbol{e}_{1}$ and $\boldsymbol{e}_{2}$ represent unit vectors along the positive $x_{1}$ - and $x_{2}$-directions, respectively.

It becomes thus understood that the implied pure bending of the plate cross-section is sustainable in polar linear elasticity only if, in addition to the traction boundary conditions depicted in Figure 1, a couple-traction having constant magnitude $\hat{m}_{3}$ and direction parallel to the $x_{3}$-axis is also applied externally on the boundaries $x_{1}= \pm L / 2$, in a point by point sense (see Figure 2 for a schematic representation).

Most interestingly, (6.4) leads further to the following definition of the fibre bending stiffness:

$$
d^{f}=\alpha\left(\frac{\hat{m}_{3}}{\hat{\sigma}_{1}}\right), \quad \alpha=C_{11}-C_{12}^{2} / C_{22}
$$

Accordingly, the value of the fibre bending stiffness $d^{f}$ is linearly proportional to the ratio $\hat{m}_{3} / \hat{\sigma}_{1}$ of the intensities of the externally applied boundary couple-traction and traction distributions. Alternatively, with use of (5.8), (6.6) is converted into the following:

$$
d^{f}=\beta\left(\frac{\hat{m}_{3}}{M_{3}}\right), \quad \beta=\frac{\alpha h^{3}}{12}=\frac{h^{3}\left(C_{11} C_{22}-C_{12}^{2}\right)}{12 C_{22}},
$$

which shows that the fibre bending stiffness is proportional to the ratio of the boundary coupletraction and the boundary bending moment resultant that make the implied plate deformation sustainable. Both proportionality factors, $\alpha$ and $\beta$, depend on the values of the elastic moduli of the corresponding non-polar fibrous composite, which are considered known.

The effective bending moment resultant which is applied externally on the edges of the polar fibre-reinforced plate is thus still parallel to the $x_{3}$-axis, while its magnitude, 


$$
M_{3}^{p}=M_{3}+h \hat{m}_{3}=h\left\{\frac{h^{2}\left(C_{11} C_{22}-C_{12}^{2}\right)}{12 C_{22}}+d^{f}\right\} \kappa_{1},
$$

is still proportional to the resulting curvature. The value of the fibre bending stiffness that characterises the polar material of interest may accordingly be estimated as follows:

$$
d^{f}=\frac{M_{3}^{p}}{h \kappa_{1}}-\frac{h^{2}\left(C_{11} C_{22}-C_{12}^{2}\right)}{12 C_{22}} .
$$

Potential use of this formula requires from the values of $M_{3}^{p}$ and $\kappa_{1}$ to become measurable, either by designing some relevant experimental set up or by appropriately refining a relevant set up that may be already available for corresponding non-polar material behaviour (e.g., Bigoni, 2010, 2011).

It is emphasised that, although the fibres of this transverse isotropic plate resist bending, (6.8) is still in agreement with the classical Euler-Bernoulli rule. Accordingly, and because $e_{12}=0$ is still valid, the outlined polar elasticity solution suggests that elements which are initially parallel to the $x_{2}$ axis remain approximately straight and normal to the deformed $x_{1}$-axis.

\subsection{Orthotropy}

In the case of special orthotropy, where two mutually orthogonal families of straight fibres reinforce the plate along the $x_{1}$ - and $x_{2}$-directions, interest is focused only in cases that the fibres of both families resist bending. This is because, as is already mentioned, the stress constitutive equation is still of the form (6.1). It is accordingly seen that, if one of the two families is made of fibres resistant in bending while the other is made of perfectly flexible fibres, this polar linear elasticity problem becomes mathematically equivalent to its transverse isotropic counterpart discussed in section 6.1.

Under these considerations, connection of the couple-stress constitutive equation (2.14) with the curvature-strain components (6.3) makes evident that, in this case, the set of displacements (5.9) produces the single nonzero couple-stress component

$\bar{m}_{13}=\left(-d_{31} S_{11}+d_{32} S_{12}\right) \hat{\sigma}_{1}=-d^{f_{1}} \kappa_{1}=-\hat{m}_{3}$,

where

$$
d^{f_{1}}=\left(d_{31}+d_{32} \frac{C_{12}}{C_{22}}\right) \text {. }
$$


Expression (6.10a) resembles closely its counterpart obtained in the preceding section and enables the analysis that follows (6.4) to be repeated on an essentially step by step basis, thus leading to

$$
d^{f_{1}}=\frac{M_{3}^{p}}{h \kappa_{1}}-\frac{h^{2}\left(C_{11} C_{22}-C_{12}^{2}\right)}{12 C_{22}} .
$$

However, there is still a significant difference between (6.10) and (6.4). Unlike $d^{f}$ which is the only fibre bending stiffness modulus appearing in (2.13), $d^{f_{1}}$ emerges here as a corresponding effective elastic modulus of fibre bending resistance, in the sense that it depends on a pair of its seven, apparently independent counterparts encountered in (2.14). Even if $d^{f_{1}}$ becomes available experimentally in the matter implied after (6.9), (6.10b) can only be regarded as a single relationship that holds between the elastic moduli $d_{31}$ and $d_{32}$.

It follows that determination of $d_{31}$ and $d_{32}$ may not become possible in the present case before a second relationship between these moduli is sought and found, along with a relevant feasible experimental set up. By generalising this observation, one may alternatively suggest that characterisation studies of the present polar orthotropic material may not be complete until a sufficient number of additional relationships that relate all seven fibre bending moduli appearing in (2.14) are sought and found. Such additional relationships may be sought in future relevant investigations that involve study not only of plane strain, but also of three-dimensional fundamental bending deformations of orthotropic polar material plates.

In this context, it is appropriate to further note that a relationship of the form (6.11) can become available between $d_{13}$ and $d_{23}$ by considering the alternative pure bending problem implied in Figure 3. This Figure depicts the cross-section of the plate implied in Figure 1, but considers that the external loading that causes pure bending is now applied on the cross-sectional edges $x_{2}= \pm h / 2$.

It follows that the prevailing stress is now

$\sigma_{22}=\hat{\sigma}_{2} x_{1}, \quad \sigma_{11}=\sigma_{(12)}=\sigma_{[12]}=0$,

where the known positive constant $\hat{\sigma}_{2}$ represents the intensity of the depicted boundary traction distributions at $x_{2}= \pm h / 2$. The exact solution of the pure bending problem implied in Figure 3 is thus completed in the manner detailed earlier in Section 5. This leads to the displacement distributions

$u_{1}=-\frac{\hat{\sigma}_{2}\left(C_{12} x_{1}^{2}+C_{11} x_{2}^{2}\right)}{2\left(C_{11} C_{22}-C_{12}^{2}\right)}, \quad u_{2}=\frac{\hat{\sigma}_{2} C_{11} x_{1} x_{2}}{C_{11} C_{22}-C_{12}^{2}}$,

and, hence, yield the following non-zero curvature-strain components: 


$$
u_{2,12}=\frac{\hat{\sigma}_{2} C_{11}}{C_{11} C_{22}-C_{12}^{2}}, \quad u_{1,11}=-\frac{\hat{\sigma}_{2} C_{12}}{C_{11} C_{22}-C_{12}^{2}}, \quad u_{1,22}=-\frac{\hat{\sigma}_{2} C_{11}}{C_{11} C_{22}-C_{12}^{2}}
$$

The magnitude of the resultant bending moment applied along the $x_{3}$-axis per unit width of the non-polar counterpart of the plate is now

$$
\tilde{M}_{3}=\left.\int_{-L / 2}^{L / 2} \sigma_{22}\right|_{x_{2}= \pm h / 2} d x_{1}=\frac{L^{3} \hat{\sigma}_{2}}{12}=\frac{L^{3}\left(C_{11} C_{22}-C_{12}^{2}\right)}{12 C_{11}} \kappa_{2}
$$

where

$$
\kappa_{2}=\left|u_{1,22}\right|=\frac{C_{11} \hat{\sigma}_{2}}{C_{11} C_{22}-C_{12}^{2}} .
$$

Because fibres resist bending, the couple-stress constitutive equation (2.14) incorporates further into the analysis the single couple-stress component

$$
\bar{m}_{23}=\frac{d_{13} C_{12}+d_{23} C_{11}}{C_{11} C_{22}-C_{12}^{2}} \hat{\sigma}_{2}=d^{f_{2}} \kappa_{2}=\tilde{m}_{3}
$$

where, in a close connection with (6.4) and (6.10), the introduced positive parameter $\tilde{m}_{3}$ represents the intensity of $\bar{m}_{23}$.

In this context, (2.4b) makes evident that the following sets of boundary couple-tractions need also to be included in the analysis:

at $x_{1}= \pm L / 2: L_{i}^{\left(e_{1}\right)}=0$,

at $x_{2}= \pm h / 2: L_{3}^{\left(e_{2}\right)}=\tilde{m}_{3}, L_{1}^{\left(e_{2}\right)}=L_{2}^{\left(e_{2}\right)}=0$.

Accordingly, the implied pure bending of the plate cross-section is sustainable in polar linear elasticity only if, in addition to the traction boundary conditions depicted in Figure 3, a coupletraction, having constant magnitude $\tilde{m}_{3}$ and direction parallel to the $x_{3}$-axis, is applied externally on the boundaries $x_{2}= \pm h / 2$ (see Figure 4 for a schematic representation).

The analysis resembles then closely its counterpart detailed after (6.5) in the preceding Section thus leading to

$$
d^{f_{2}}=\left(d_{23}+d_{13} \frac{C_{12}}{C_{11}}\right)=\frac{\tilde{M}_{3}^{p}}{h \kappa_{2}}-\frac{L^{2}\left(C_{11} C_{22}-C_{12}^{2}\right)}{12 C_{11}},
$$

where, as is mentioned after (6.9), the values of $\kappa_{2}$ and $\tilde{M}_{3}^{p}$ may also be considered potentially measurable. In the light of the previous relevant discussion, determination of $d_{23}$ and $d_{13}$ may not 
become possible before a second relationship between these moduli is sought and found. Alternatively, (6.11) and (6.19) may be considered as two of the aforementioned larger number of relationships required for complete determination of the fibre bending moduli appearing in (2.14).

\section{Conclusions}

It has become clear in Section 5 that the equations of conventional, non-polar linear elasticity of fibre-reinforced materials are essentially not able to distinguish between plane strain bending behaviour of transverse isotropic and special orthotropic plates. Another case, in which non-polar linear elasticity fails to even detect the presence of perfectly flexible fibres has already been identified in (Dagher and Soldatos, 2011), while other similar cases may appear in the future.

In contrast, polar material considerations stemming from fibres ability to resist bending enable linear elasticity to properly account for the presence of fibres and, hence, to make appropriate distinctions between transverse isotropy and special orthtropy. It is interesting thought that, in either case, polar linear elasticity reveals that, if a family of fibres resistant in bending is parallel to the $x_{1}$ or the $x_{2}$-axis, then the bending deformation implied in Figures 1 or 3 is sustainable only if, in addition to the depicted traction boundary conditions, a certain constant couple-traction is also applied externally on the same boundaries (see Figures 2 and 4, respectively).

Invention of methods appropriate for characterisation of fibre-reinforced materials that exhibit this kind of polar material behaviour presents thus immense interest and purpose. In the case of transverse isotropy due to a single family of fibres resistant in bending, it is accordingly found that, if accompanied by a relevant carefully designed experimental set up, the pure bending plate problem employed in this investigation can enable identification of the single fibre bending stiffness that enters the analysis. Nevertheless, in the corresponding case of special orthotropy where use is made of seven fibre bending stiffnesses, the implied pure bending problem seems currently unable to connect the latter with more than two relevant relationships.

It thus follows that additional such relationships are seemingly required to be sought in the case of orthotropic polar material behaviour, possibly through identification and solution of corresponding fundamental problems of three-dimensional plate bending. In such cases though, 
potential consequences of the non-elliptic nature of the governing equations of polar linear elasticity should also be carefully attended to. This is because, unless the relevant couple-stress field is constant throughout the plate span, the non-elliptic nature of those equations does not necessarily guarantee uniqueness of their solution

It is recalled in this context that the polar material extension of Clapeyron's theorem detailed in Section 4 enabled this investigation to reach the conclusion that, if the couple-stress field generated during a small deformation a fibre-reinforced material is constant, then the solution of the corresponding polar linearly elasticity boundary value problem is unique. The pure bending deformation of polar fibre-reinforced materials considered this study does generate such a constant couple stress-field and, hence, the corresponding polar elasticity solutions demonstrated and used in Section 6 are indeed unique.

\section{References}

1. Adkins, J.E. (1951) Studies in the Theory of Large Elastic Deformations. A Thesis presented for the Degree of Ph.D, London University.

2. Adkins, J.E. and Rivlin, R.S. (1955) Large elastic deformations of isotropic materials X. Reinforcement by inextensible cords. Philos. Trans. R. Soc. London A 248, 201-223.

3. Adkins, J.E. (1956) Finite plane deformations of thin elastic sheets reinforcement with inextensible cords. Philos. Trans. R. Soc. London A 249, 125-150.

4. Bhimaraddi, A. and Stevens, L.K. (1984) A higher order theory for free vibration of orthotropic, homogeneous and laminated rectangular plates. J. Appl. Mech. 51, 195-198.

5. Dagher, M.A. and Soldatos, K.P. (2011) On small azimuthal shear deformation of fibre-reinforced cylindrical tubes. J. Mech. Mater. Struct. 6, 141-168.

6. Donnell, L.H. (1976) Beams, Plates and Shells. McGraw-Hill, New York.

7. Dorfmann, A., Merodio J. and Ogden, R.W. (2010) Non-smooth solutions in the azimuthal shear of an anisotropic nonlinearly elastic material. J. Eng. Math. 68, 27-36

8. El Hamdaoui, M. and Merodio, J. (2015) Azimuthal shear of doubly fibre-reinforced, non-linearly elastic cylindrical tubes. J. Eng. Math. 95, 347-357. 
9. Jones, R.M., Mechanics of Composite Materials, Taylor \& Francis, Washington (1998).

10. Kirchhoff, G. (1850) Über das Gleichgewicht und die Bewegung einer elastischen Scheibe. J. Reine Angew Math. 40, 51-58.

11. Knowles, J.K. and Sternberg, E. (1975) On the ellipticity of the equations of nonlinear elastostatics for a special material. J. Elasticity 5, 341-361

12. Love, A.E.H. (1944) A Treatise on the Mathematical Theory of Elasticity. Reprint Ed., Dover Publ., New York.

13. Merodio, J. and Ogden, R.W. (2002) Material instabilities in fiber-reinforced non-linearly elastic solids under plane deformation, Arch. Mech. 54, 525-552.

14. Merodio, J. and Ogden, R.W. (2003) Instabilities and loss of ellipticity in fiber-reinforced compressible non-linearly elastic solids, Int. J. Solids Struct. 40, 4707-4727.

15. Mindlin, R.D. (1951) Influence of rotatory inertia and shear on flexural motions of isotropic, elastic plates. ASME Trans. J. Appl. Mech. 18, 31-38.

16. Pipkin, A.C. and Rogers, T.G. (1971) Plane deformations of incompressible fiber-reinforced materials. J. Appl. Mech., Trans. ASME 38: 634-640.

17. Reddy, J.N. (1984) A simple higher-order theory for laminated composite plates. J. Appl. Mech. 51, 745-752.

18. Reissner, E. (1945) The effect of transverse shear deformation on the bending of elastic plates. ASME Trans. J. Appl. Mech. 12, 69-77.

19. Reissner, E. and Stavsky, Y. (1961) Bending and stretching of certain types of heterogeneous aeolotropic elastic plates. ASME J. Appl. Mech. 28, 402-408.

20. Rivlin, R.S. (1955) Plane strain of a net formed by inextensible cords. J. Ration. Mech. Anal. 4, 511-535.

21. Roccabianca, S., Gei, M. and Bigoni, D. (2010) Plane strain bifurcations of elastic layered structures subject to finite bending: theory versus experiments. IMA J. Appl. Math. 75, 525-548.

22. Roccabianca, S., Bigoni, D. and Gei, M. (2011) Long wavelength bifurcations and multiple neutral axes of elastic layered structures subject to finite bending. J. Mech. Mater. Struct. 6, 510527.

23. Sokolnikoff, I.S. (1983) Mathematical Theory of Elasticity. Reprint Ed., Krieger, Malabar, Florida. 
24. Soldatos, K.P. (2003) Accurate stress analysis of laminated composite components, in "Modern Trends in Composite Laminates Mechanics", A. Altenbach and W. Becker, eds, Springer Verlag, CISM Courses and Lectures no 448 (ISBN 3-211-20302-8), pp. 69-132, 2003.

25. Soldatos, K.P. (2014) Foundation of polar linear elasticity for fibre-reinforced materials. J. Elast. 114, 155-178.

26. Soldatos, K.P. (2015) Foundation of polar linear elasticity for fibre-reinforced materials II: Advanced anisotropy. J. Elast. 118, 223-242.

27. Soldatos, K.P. and Watson, P. (1997) A method for improving the stress analysis performance of two-dimensional theories for composite laminates, Acta Mech. 123, 163-186.

28. Spencer, A.J.M. (1972) Deformations of Fibre-Reinforced materials. Clarendon Press, Oxford.

29. Spencer, A.J.M., ed., (1984) Continuum Theory of the Mechanics of Fibre-reinforced Materials. CISM Courses and Lectures no 282, Springer Verlag, Wien - New York.

30. Spencer, A.J.M. (2009) Ronald Rivlin and invariant theory. Int. J. Engng Sci. 47, 1066-1078.

31. Spencer, A.J.M. and Soldatos, K.P. (2007) Finite deformations of fibre-reinforced elastic solids with fibre bending stiffness. Int. J. Non-lin. Mech. 42, 355-368.

32. Timoshenko, S.P. (1921) On the correction for shear of the differential equation for transverse vibration of prismatic bars. Phil. Magazine 41, 744-746.

33. Timoshenko, S.P. (1922) On the transverse vibration of bars of uniform cross-section. Phil. Magazine 43, 125-131.

34. Timoshenko, S.P. and Goodier, J.N. (1970) Theory of Elasticity. $3^{\text {rd }}$ Ed., McGraw-Hill, New York.

35. Ting, T.C.T., Anisotropic Elasticity. Oxford University Press, New York (1996).

36. Triantafyllidis, N. and Abeyarante, R. (1983) Instabilities of a finitely deformed fibrereinforced elastic material. J. Appl. Mech. 50, 149-156 


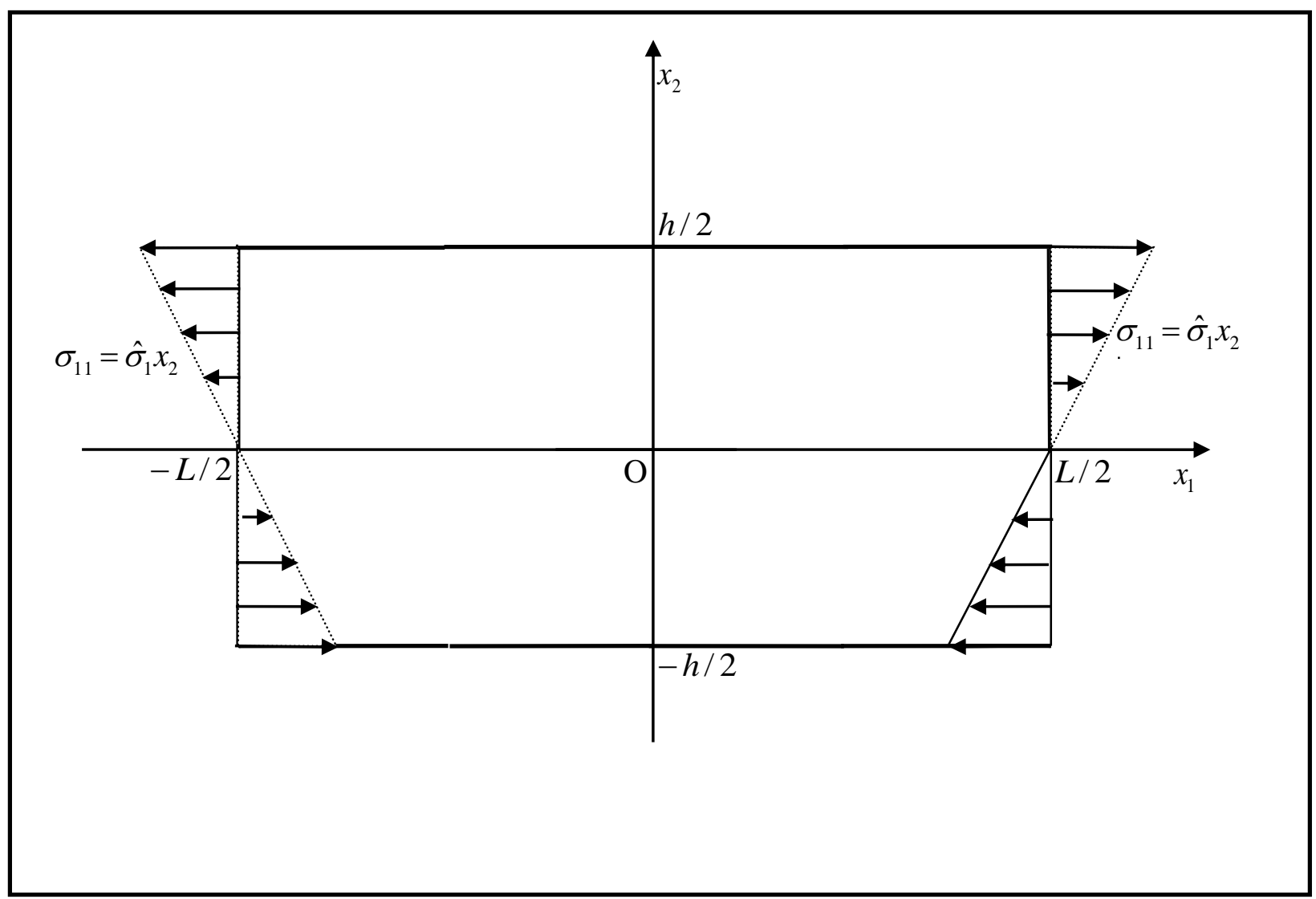

FIGURE 1: Schematic representation of the externally applied boundary traction distributions that create pure (plane strain) bending of the cross-section of a non-polar linearly elastic rectangular plate having infinite length in the $x_{3}$-direction. 


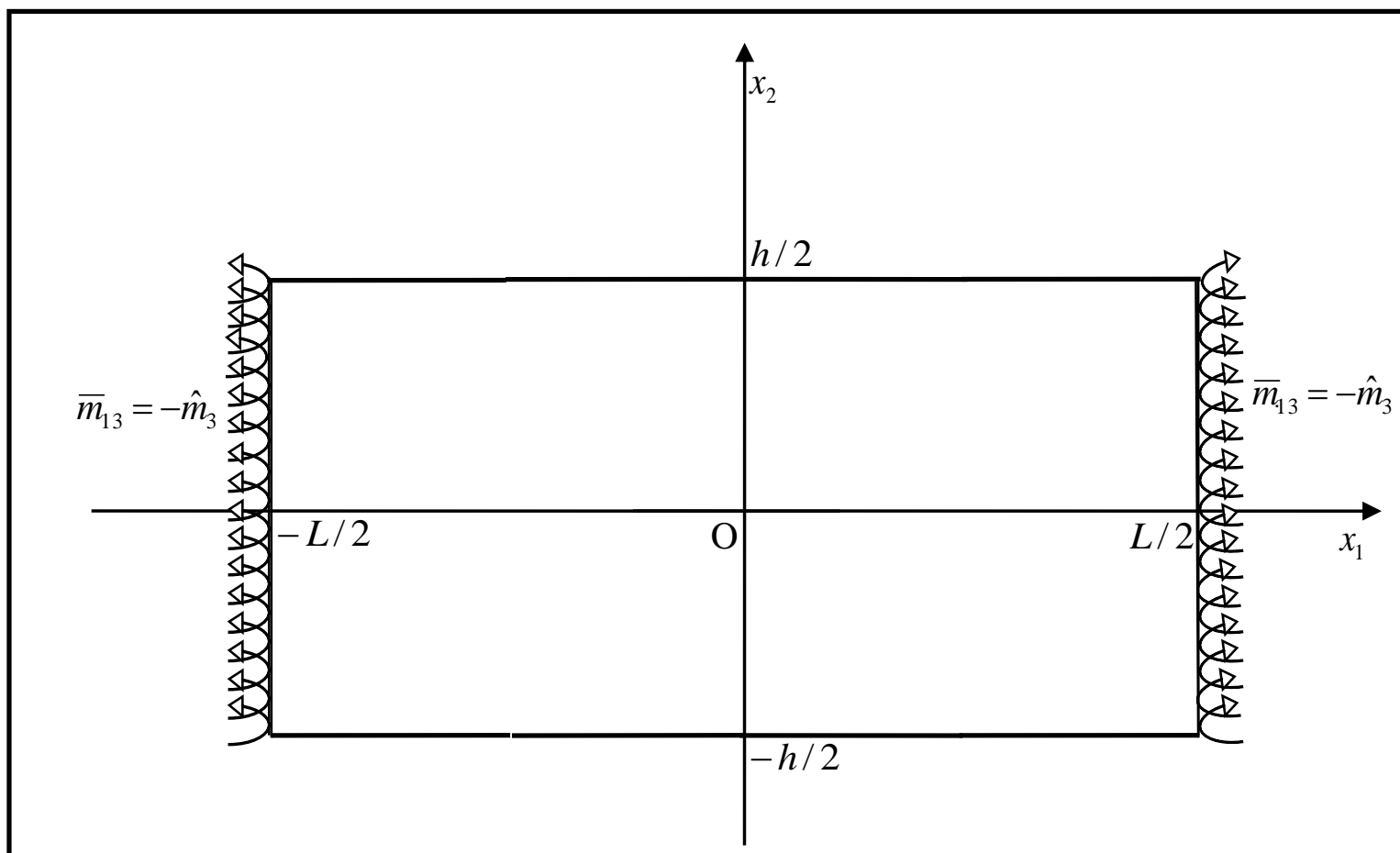

FIGURE 2: Schematic representation of constant couple-traction distributions which are superposed on the externally applied loading depicted in Figure 1, for a corresponding polar iibre-reinforced elastic plate to maintain the implied pure bending deformation. 


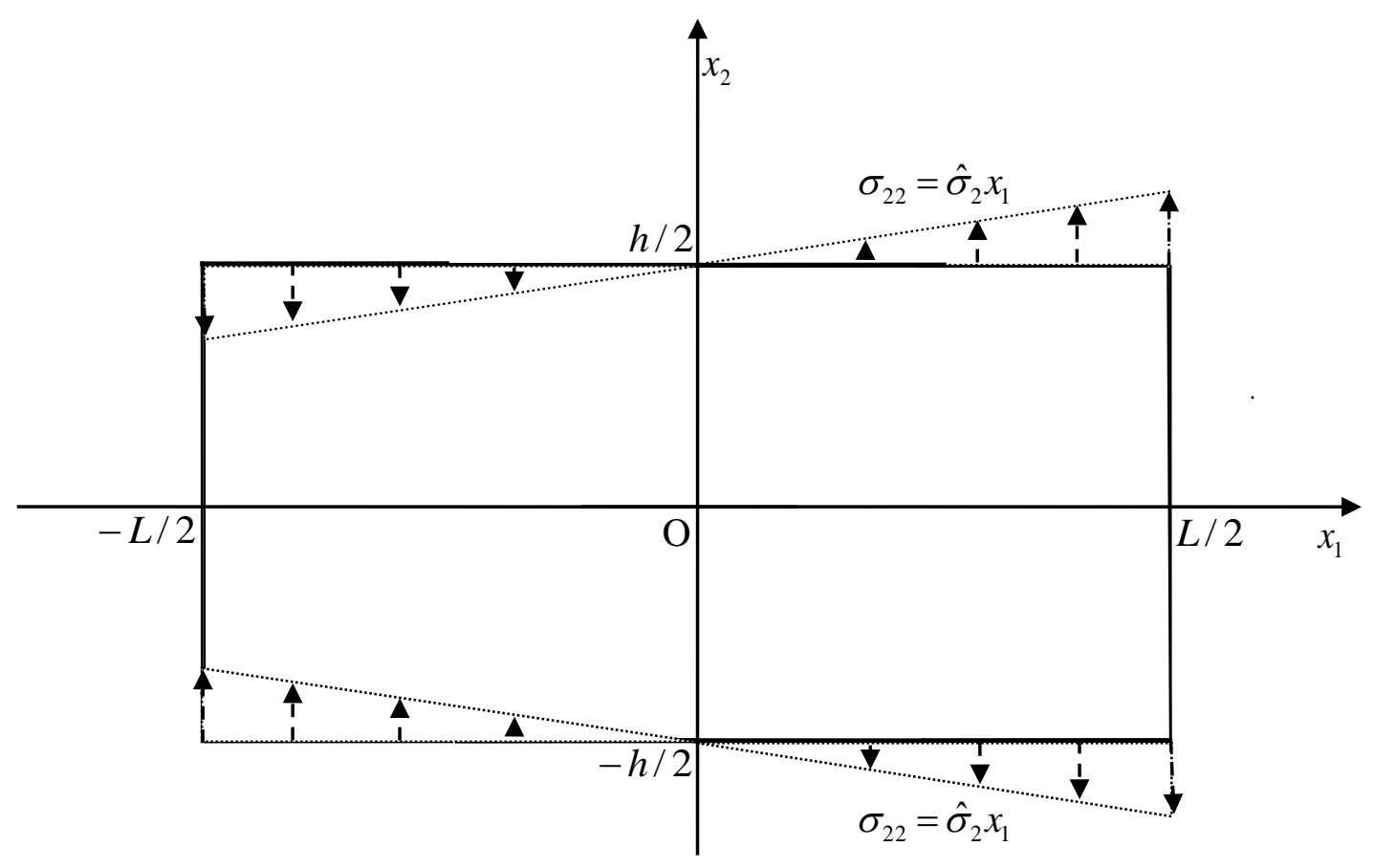

FIGURE 3: Schematic representation of the alternative pure bending deformation mode of the crosssection of the non-polar linearly elastic plate depicted in Figure 1.

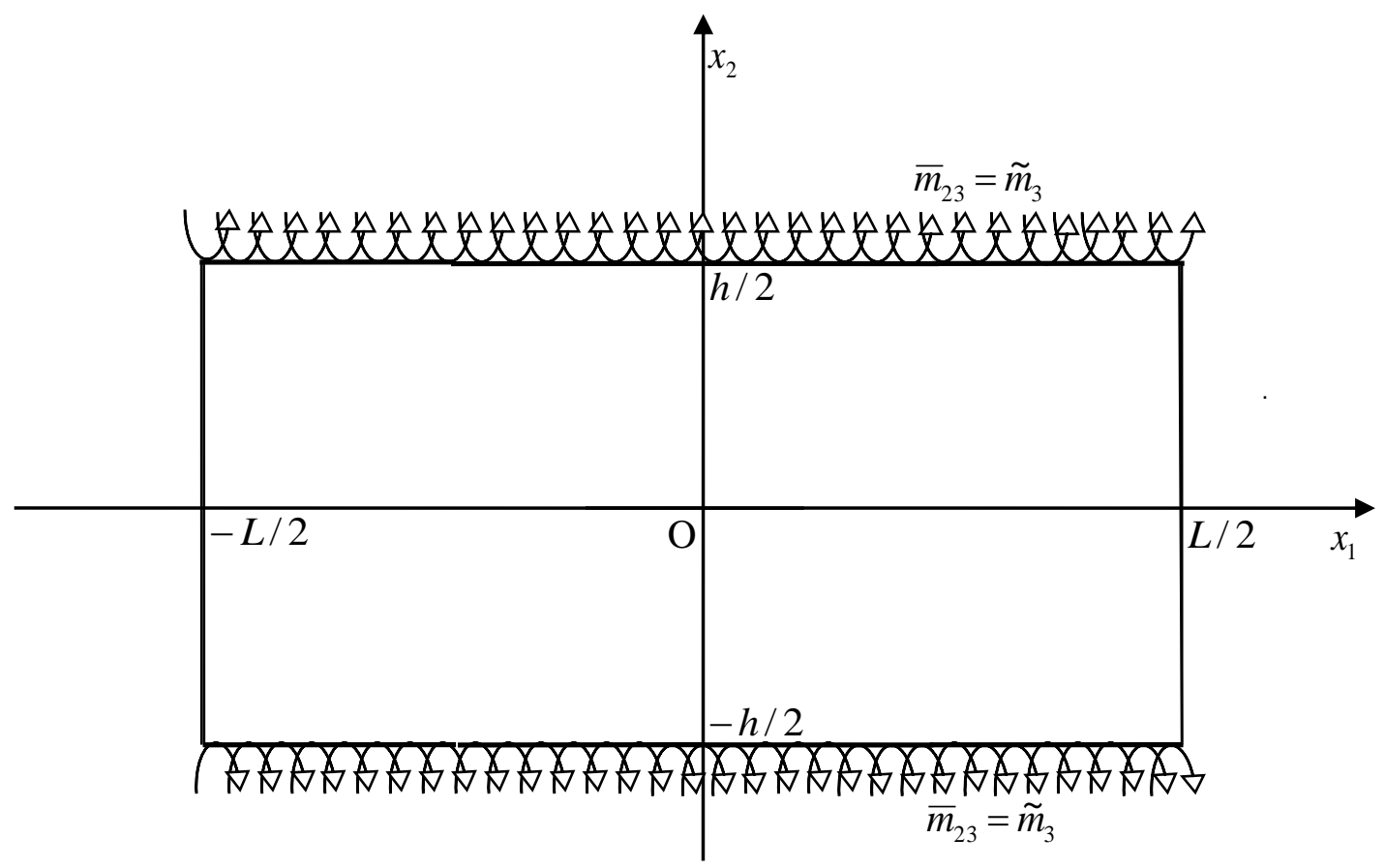


FIGURE 4: Schematic representation of constant couple-traction distributions which are superposed on the externally applied loading depicted in Figure 3, for a corresponding polar fibre-reinforced elastic plate to maintain the implied pure bending deformation. 